\title{
Gap Junctional Intercellular Communication Increases Cytotoxicity and Reduces Resistance to Hydroxyurea
}

\author{
Randall J. Ruch1*, Paul D. Boucher², Brian G. Gentry²,3, Donna S. Shewach ${ }^{2}$ \\ ${ }^{1}$ Department of Biochemistry and Cancer Biology, University of Toledo, Toledo, USA \\ ${ }^{2}$ Department of Pharmacology, University of Michigan, Ann Arbor, USA \\ ${ }^{3}$ Drake University College of Pharmacy and Health Sciences, Des Moines, USA \\ Email: "randall.ruch@utoledo.edu
}

Received 29 August 2014; revised 25 September 2014; accepted 20 October 2014

Copyright (C) 2014 by authors and Scientific Research Publishing Inc.

This work is licensed under the Creative Commons Attribution International License (CC BY). http://creativecommons.org/licenses/by/4.0/

(c) (7) Open Access

\begin{abstract}
Background: Gap junctions enable small molecules to diffuse between adjacent cells and have been associated with greater cytotoxicity of radiation and anti-cancer drugs. We investigated whether this gap junctional intercellular communication (GJIC) affected the cytotoxicity of the classic ribonucleotide reductase (RR) inhibitor and anti-cancer agent, hydroxyurea (HU). Materials and Methods: We used GJIC-proficient and deficient, connexin 43-expressing WB rat liver epithelial cell lines. We compared HU toxicity by crystal violet assay, effects of the drug on deoxynucleotide pools by HPLC, and ability of GJIC to increase toxicity of HU-resistant cells through a bystander effect in co-culture experiments. Results: GJIC-proficient cells were three- to five-fold more sensitive $\left(\mathrm{IC}_{50} 0.1 \mathrm{mM}\right)$ to $\mathrm{HU}$ than GJIC-deficient derivatives $\left(\mathrm{IC}_{50} 0.3-0.5 \mathrm{mM}\right)$. This sensitivity depended upon GJIC because treatment of GJIC-proficient cells with the GJIC blocker oleamide decreased HU toxicity by approximately $60 \%-80 \%$ and restoration of GJIC in GJIC-deficient cells by stable transduction of connexin 32-encoding $G j b 1$ increased $\mathrm{HU}$ toxicity $\left(\mathrm{IC}_{50} 0.1 \mathrm{mM}\right)$. The effects were not due to connexin expression per se or its localization since all cell lines expressed comparable quantities of connexin $\mathbf{4 3}$ that was localized to the plasma membrane. Also HU sensitivity was not related to differential effects on nucleotide metabolism in the cells. Thymidine triphosphate levels increased and deoxyadenosine triphosphate levels decreased similarly (15\% - 20\%) in GJIC-proficient and deficient cells over $24 \mathrm{~h}$ of HU treatment. More importantly, when HU-resistant cells were co-cultured with sensitive cells, the resistant cells were killed only when GJIC was present. Conclusion: The data suggest that GJIC enhances cytotoxicity and decreases resistance to HU. These results may be important clinically if GJIC can be enhanced in drug-resistant cells.
\end{abstract}

"Corresponding author.

How to cite this paper: Ruch, R.J., Boucher, P.D., Gentry, B.G. and Shewach, D.S. (2014) Gap Junctional Intercellular Communication Increases Cytotoxicity and Reduces Resistance to Hydroxyurea. Journal of Cancer Therapy, 5, 1190-1202. 


\section{Keywords}

\section{Bystander Effect, Drug Resistance, Gap Junctions, Hydroxyurea, Ribonucleotide Reductase}

\section{Introduction}

Gap junctions are plasma membrane channels that connect the interiors of neighboring cells and permit the direct cell-to-cell diffusion of molecules and ions smaller than $\sim 1 \mathrm{kDa}$. This is known as gap junctional intercellular communication (GJIC) [1]. Gap junction channels are formed by proteins known as connexins and 21 human and 20 murine connexins have been identified [2]. Two gap junction hemichannels, also known as connexons, on opposite cells dock together to form a patent gap junction channel and connect the two cell interiors. Typically hundreds to thousands of gap junction channels are aggregated in a single gap junction and several gap junctions occur between apposed cells. Thus there is a large capacity for rapid diffusion of small molecules and ions via GJIC. The physiological roles of GJIC are numerous and include cellular homeostasis; regulation of cell proliferation, death, and differentiation; coordination of cellular responses to external stimuli; and neural coupling through electrical synapses. Deficits in GJIC have been associated with many diseases including cancer, cardiac arrhythmia, teratogenesis, cataracts, atherosclerosis, and deafness. Connexins irrespective of forming gap junction channels also interact with many structural and signaling proteins and have many physiological roles that are independent of GJIC [3] [4].

The responses of cells to cytotoxic drugs and radiation are impacted by GJIC and connexins. In some cases, cytotoxicity was directly correlated with GJIC whereas reduced cell death occurred in GJIC-coupled cells [5]. The mechanisms for these effects are poorly understood. Injured cells may impact naive cells through poorly understood bystander effects whereby toxic factors and death-inducing signal molecules such as calcium ion and free radicals may move from injured cells to neighbors through gap junction channels. In contrast, protective agents such as glutathione may move from naive to injured cells and protect the latter. Connexins may also alter cell death in a non-GJIC-dependent manner through their roles in cell signaling pathways. Damaged cells may also release toxic molecules into the external milieu via gap junction hemichannels.

Ribonucleotide reductase (RR) inhibitors are an important class of anti-cancer drugs, but how GJIC affects their cytotoxicity has not been well studied. This enzyme catalyzes the de novo conversion of ribonucleotide diphosphate to deoxyribonucleoside diphosphate and is the rate-limiting step in DNA synthesis [6]. The enzyme consists of two subunits, M1 and M2, both of which are dimers. The enzyme catalytic site is located in M2 and contains a tyrosyl free radical and a non-heme iron. M1 is expressed throughout the cell cycle, but not in $\mathrm{G}_{0}$, and M2 is expressed during S phase. Pairing of M1 and M2 is necessary for RR catalytic activity during S-phase. Many inhibitors of RR have been developed and are widely used for cancer therapy. These include older drugs such as hydroxyurea (HU) and newer ones such as gemcitabine (2', 2'-difluorodeoxycytidine). Hydroxyurea is a potent inhibitor of RR and is used to treat myeloproliferative disorders such as chronic myelogenous leukemia, some solid cancers in combination with other agents or radiation, and non-hematological diseases like sickle cell anemia [7]. Treatment of proliferating cells with RR inhibitors causes an imbalance of deoxynucleotide pools, abortion of DNA replication, and the activation of endonucleases and apoptosis.

Here we have investigated whether GJIC affected the cytotoxicity of HU. We found that HU was more toxic in GJIC-positive cells and HU resistant cells were killed when co-cultured with HU-sensitive cells in a GJICdependent manner.

\section{Materials and Methods}

\subsection{Reagents}

Gentamicin sulfate, HU, oleamide, and Lucifer Yellow CH were obtained from Sigma-Aldrich (St. Louis, MO). Richter's improved minimal essential medium and G418 sulfate were purchased from Life Technologies (Grand Island, NY). Fetal bovine serum was from Atlanta Biologicals (Flowery Branch, GA). The BioRad DC protein assay was obtained from Bio-Rad (Herculaes, CA). 


\subsection{Cell Lines and Culture Medium}

All cell lines were cultured in Richter's Improved MEM supplemented with 5\% fetal bovine serum and gentamicin sulfate $(40 \mu \mathrm{g} / \mathrm{ml})$ and passaged by trypsinization. The Gjb2-transduced cells were also cultured in the presence of G418 $(0.4 \mathrm{mg} / \mathrm{ml})$ during routine culture, but not during experimental use.

\subsection{Cytotoxicity Assays}

Cytotoxicity was determined by crystal violet staining, colony formation, and trypan blue dye exclusion assays. For the crystal violet assay, cells were plated into 24 well culture dishes at densities that resulted in approximately $75 \%$ confluence, allowed to attach overnight, then treated with $\mathrm{HU}$ for $48 \mathrm{~h}$. After treatment, the culture medium was aspirated and the cells were washed and refed with fresh medium. The cells were cultured for three additional days, then fixed with $10 \%$ buffered formalin, stained with $2 \%$ crystal violet for five minutes, washed extensively to remove excess dye, and allowed to air dry. The cells were then dissolved in $1 \mathrm{ml}$ of $2 \%$ SDS and centrifuged at $10,000 \times \mathrm{g}$ for $5 \mathrm{~min}$ to remove debris. Absorbance of the supernatant was read on a spectrophotometer at $\mathrm{A}_{570}$. In some cases, oleamide, which is a potent inhibitor of GJIC mediated by Cx43 channels [8], was used to block gap junction coupling in WB cells. The concentrations and treatment durations of $\mathrm{HU}$ and oleamide are noted in the results and figure legends.

Cytotoxicity was also determined by colony formation assay. As above, approximately $75 \%$ confluent cultures in six well dishes were treated with $\mathrm{HU}$ and/or oleamide for $48 \mathrm{~h}$. Then the cells were trypsinized and plated into $60 \mathrm{~mm}$ dishes at a colony density of 200 cells per well. After $5 \mathrm{~d}$, the cells were fixed with $10 \%$ buffered formalin and stained with crystal violet. Colonies that contained at least 40 cells were counted.

\subsection{Generation of HU Resistant Cells and Toxicity of HU in Co-Cultured Sensitive and Resistant Cells}

Hydroxyurea-resistant WB-F344 and WB-aB1 cells were generated by stepwise increases in drug concentration in the culture medium, i.e., from $0.05 \mathrm{mM}$ to $2 \mathrm{mM}$ over 6 weeks after which the cells were completely resistant to the high dose of $\mathrm{HU}$. These HU-resistant lines were designated WB-F344/HU ${ }^{\mathrm{R}}$ and WB-aB1/HU .

To determine if GJIC could impact drug sensitivity in these HU resistant cells, HU sensitive and GJIC-proficient WB-F344 or deficient WB-aB1 cells were co-cultured with GJIC-proficient WB-F344/HU ${ }^{\mathrm{R}}$ or deficient $\mathrm{WB}-\mathrm{aB} 1 / \mathrm{HU}^{\mathrm{R}}$ cells and treated with $1 \mathrm{mM}$ HU for $48 \mathrm{~h}$. The cells were co-cultured at a 3:1 (sensitive:resistant) ratio to increase the likelihood that HU-resistant cells would contact sensitive cells. The resistant cells were also pre-labeled with PKH-26, a vital fluorescent plasma membrane dye [9] in order to distinguish them from sensitive cells. After $2 \mathrm{~d}$ treatment with $\mathrm{HU}$, remaining cells were trypsinized, stained with trypan blue to identify viable cells, and counted with a hemocytometer.

\subsection{Determination of Deoxynucleotide Content in WB Cells}

Deoxynucleotides in control and HU-treated WB cells were quantified by anion exchange HPLC as described [10].

\subsection{Assessment of GJIC}

GJIC was assessed in WB cells by scrape-loading/dye transfer (SL/DT) [11] [12]. Cells were cultured to confluence in six well dishes then fluorescent Lucifer Yellow $\mathrm{CH}$ was added from stock solution (5\% w/v in PBS) to a final concentration of $0.05 \%$. Three linear cuts across the monolayer were made with a scalpel blade. Five minutes later, the medium was aspirated and the cells washed three times with serum free medium and fixed with $10 \%$ buffered formalin. At five randomly selected points along each of the three cut lines, fluorescent cells perpendicular to the cut were counted on both sides of the line (cells directly on the cut edge were not counted to exclude the dye-loaded cells). The mean of these 30 cell counts was the SL/DT index for that culture.

\subsection{Western Blotting}

The contents of connexin 43, connexin 32, and the M2 subunit of RR in cells were determined by western blotting [13]. For the connexins, samples were prepared by lysis in $1 \mathrm{ml}$ of ice cold hypotonic buffer (10 mM Tris, 
pH 7.5, 1 mM iodoacetamide, $2 \mathrm{mM}$ PMSF), followed by alkalinization of the samples by the addition of 0.33 $\mathrm{ml}$ of $40 \mathrm{mM} \mathrm{NaOH}$, sonication, and centrifugation at 13,000 rpm for $30 \mathrm{~min}$ at $4^{\circ} \mathrm{C}$. The pellets were washed once and dissolved in 2\% SDS. Then the protein content was determined (Bio-Rad DC protein assay; Bio-Rad Corp., Richmond, CA) and $20 \mu$ g of protein per sample were run on 10\% SDS-PAGE gels and blotted to Immobilon-P membranes (Millipore Corp., Bedford, MA). Connexin 43 and connexin 32 were detected using mouse monoclonal antibodies from Zymed Corp. (South San Francisco, CA) followed by a biotin-streptavidin alkaline phosphatase secondary detection kit with 5-bromo-4-chloro-3-indolyl-phosphate/nitrobluetetrazoliumcolor development (Amersham Corp., Arlington Heights, IL). For RR-M2, the samples were prepared by lysis in $1 \mathrm{ml}$ of ice cold RIPA buffer (25 mM Tris, pH 7.6, $150 \mathrm{mM} \mathrm{NaCl}, 1 \% \mathrm{NP}-40,1 \%$ sodium deoxycholate, $0.1 \%$ SDS, and $2 \mathrm{mM}$ PMSF) followed by sonication, centrifugation, and determination of protein content in the supernatants as above. Samples ( $25 \mu \mathrm{g}$ of protein) were run on 10\% SDS-PAGE gels and blotted to Immobilon-P membranes. The RR-M2 was detected with anti-RRM2 goat polyclonal antibody from Santa Cruz Biotechnology, Inc. (Dallas, TX) and secondary detection as above.

\subsection{Cell Proliferation}

The proliferation rates of the cell lines were determined as the population doubling times. Briefly cells were plated at low density $\left(2.5 \times 10^{4}\right.$ cells/well in 24 well dishes), then trypsinized and counted over the next 12 days. The PDT was determined from the log phase growth phase over the first 4 - 6 days of culture [14].

\section{Results}

\subsection{GJIC and Connexin Expression in WB Cell Lines}

Several WB cell lines were used in this study. WB-F344 is a nontumorigenic, diploid, rat liver epithelial cell line [15] that exhibits extensive GJIC and expresses the gap junction protein connexin 43 (Cx43) in localized punctata on the plasma membrane between apposed cells as expected for gap junctions (Figures 1(a)-(c)). Several GJIC-deficient derivatives of WB-F344 [16] and known as WB-aB1, WB-bA2, WB-cD6, and WB-dA2 were also used here. These cells do not exhibit GJIC by SL/DT assay, but express Cx43 in amounts comparable to WB-F344 cells; this protein is also localized to the plasma membrane (Figures 1(a)-(c)). In addition, GJIC-deficient WB-aB1 cells that were stably transduced with Gjb1 which encodes connexin 32 (Cx32) [17] were used. Two of the clones, WB-a/32-9 and WB-a/32-10, exhibit levels of dye-coupling similar to WB-F344 cells and express Cx32 (Figures 1(a)-(c)). The population doubling times of these cell lines were determined (Figure 1(d)). Notably, the PDTs did not correlate with GJIC or the type of connexin expressed.

\subsection{HU Cytotoxicity in GJIC-Proficient and GJIC-Deficient WB Cells}

The toxicity of $\mathrm{HU}$ in the above cell lines was determined by crystal violet staining of the cultures after $48 \mathrm{~h}$ exposure to the drug and 3 d subsequent growth in the absence of the compound. GJIC-proficient WB-F344, WB-a/32-9, and WB-a/32-10 cells were more sensitive to HU than the other GJIC-deficient cells. Much more crystal violet staining was evident in GJIC-deficientcell cultures (Figure 2(a)). Quantification of the crystal violet after dissolution of the stained cells and spectrophotometry revealed dose-response that correlated with a leftward shift in the dose response curve for GJIC-proficient cells (Figure 2(b)). From these plots, the IC $\mathrm{C}_{50}$ values for HU in each cell line were approximated. These values were $0.1 \mathrm{mM}$ for GJIC-proficient WB-F344, WB-a/32-9, and WB/a32-10 cells and 0.3, 0.3, 0.3, and 0.5 for WB-aB1, WB-bA2, WB-dA2, and WB-cD6, respectively.

These $\mathrm{IC}_{50}$ values did not correlate with the proliferation rates of the cell lines (Figure 1(d)). For example, WB-cD6 cells were the least sensitive to $\mathrm{HU}$ with an $\mathrm{IC}_{50}$ of $0.5 \mathrm{mM}$ and one of the longest PDTs at $19.0 \mathrm{~h}$.

\subsection{Effect of a GJIC Inhibitor of HU Cytotoxicity}

We next used the GJIC inhibitor, oleamide, to determine whether the greater sensitivity of GJIC-proficient cells to HU was dependent upon GJIC. Oleamide rapidly shuts down $\mathrm{Cx} 43$ gap junction channels without affecting its expression [8]. We treated WB-F344 or WB-aB1 cells with HU at approximate $\mathrm{IC}_{75}$ concentrations $(0.125$ and $0.5 \mathrm{mM}$, respectively) for $48 \mathrm{~h}$ in the presence or absence of oleamide $(50 \mu \mathrm{M})$ then determined cytotoxicity by 


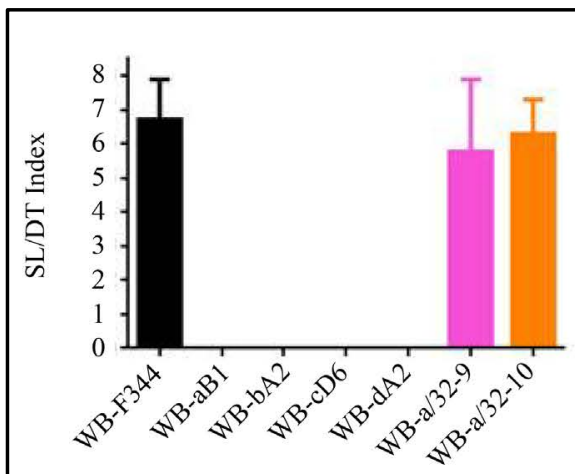

(a)

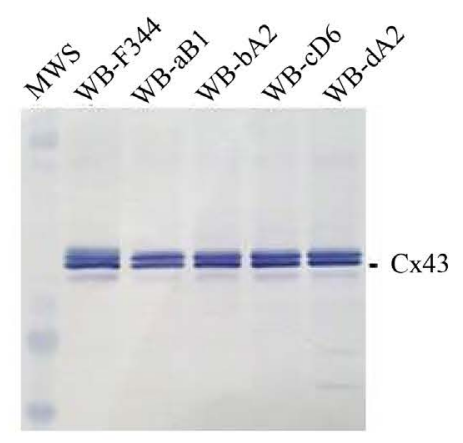

(c)
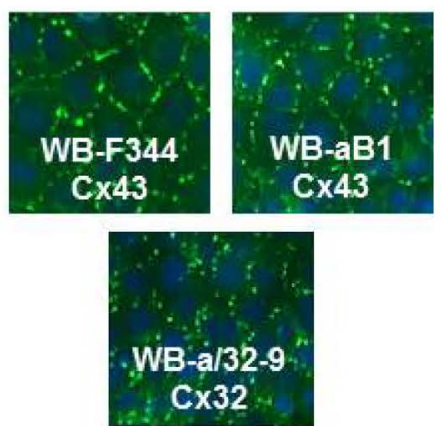

(e)
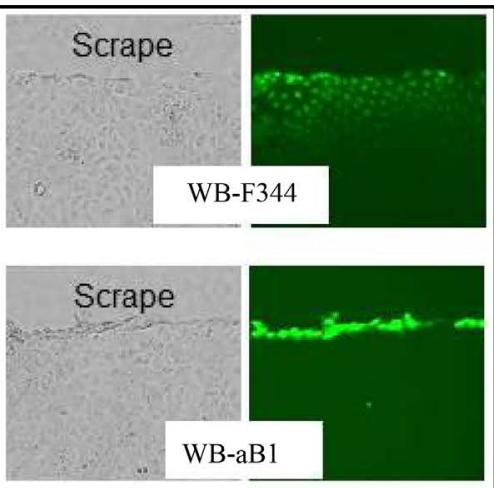

(b)

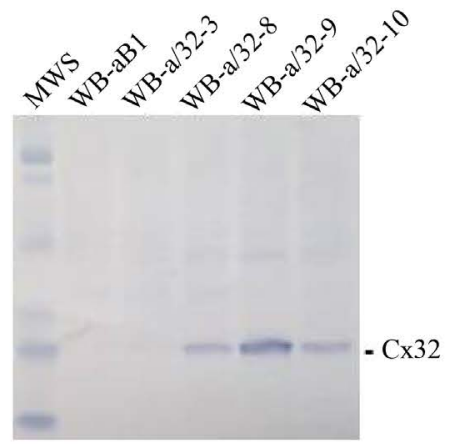

(d)

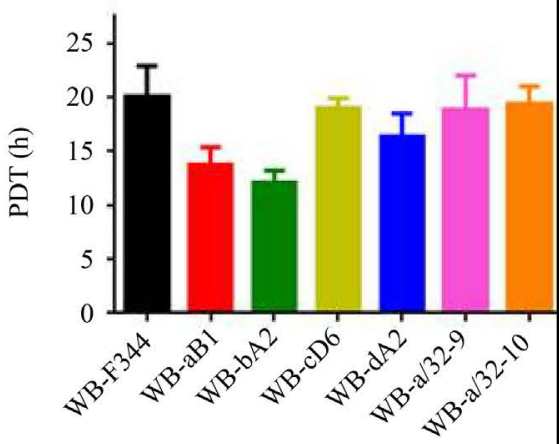

(f)

Figure 1. Gap junctional intercellular communication (GJIC), connexin expression, and cell proliferation in WB cell lines. Panel (a) illustrates levels of GJIC in the cell lines determined by the scrape-loading/dye transfer (SL/DT) assay. The bars indicate the mean \pm S.D. $(n=4$ cultures per bar). In panel (b), GJIC-proficient WB-F344 cells and GJIC-deficient WB-aB1 cells can be seen after SL/DT. Panel (c) and (d) show western blots of connexin 43 or connexin 32 in these cell lines; WB-a/32-3, -8, -9, and -10 are clones of WB-aB1 cells isolated after transduction with Gjb1. Panel (e) illustrates representative connexin 43 and connexin 32 immunostaining in the cells. In panel (e), the cell proliferation rates of the cell lines was determined as their population doubling time (PDT). The bars indicate the mean \pm S.D. $(n=3)$.

crystal violet staining (Figure 3(a)) and colony formation assay (Figure 3(b)). We used IC 75 concentrations in order to increase the likelihood of seeing a protective effect of oleamide. This compound in fact reduced the toxicity of HU, but only in GJIC-proficient WB-F344 cells and not in deficient WB-aB1 cells (Figure 3(a), 


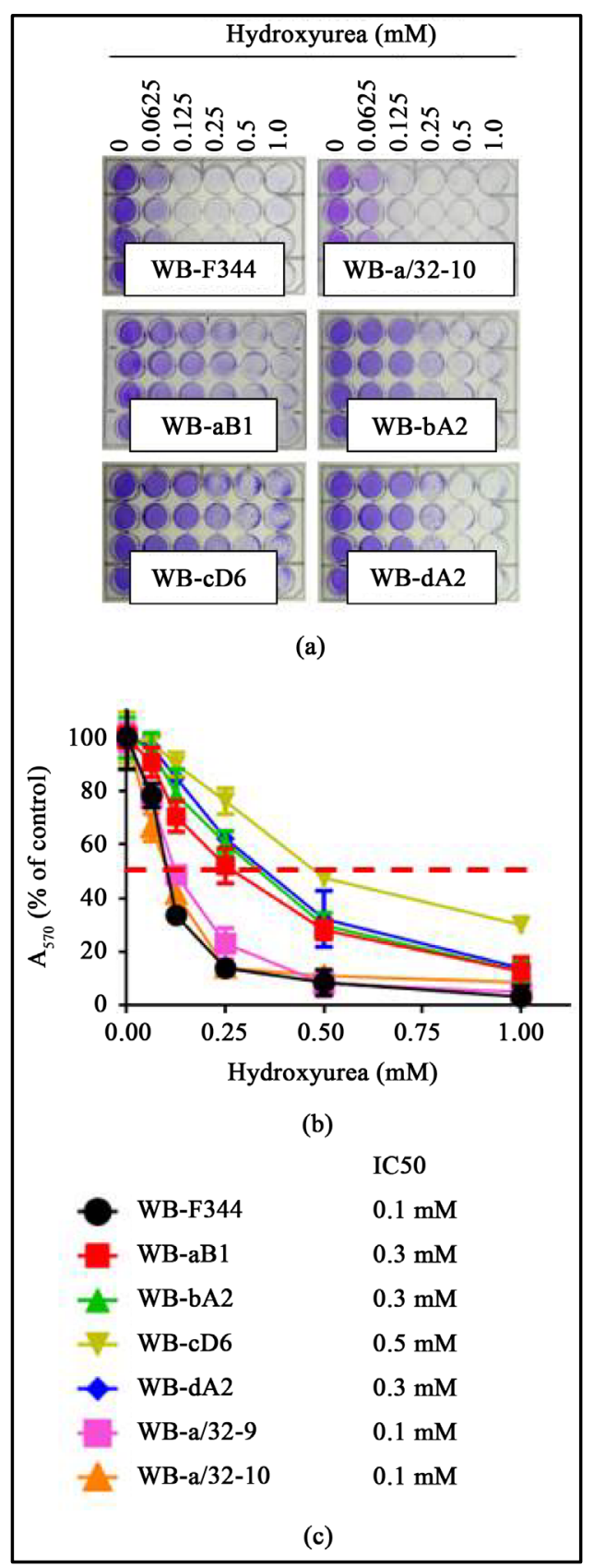

Figure 2. Cytotoxicity of HU in WB cell lines. Panel (a) illustrates the cell cultures after treatment with hydroxyurea and staining with crystal violet. In panel (b), this staining was quantified by solubilizing the residual cells in $2 \%$ SDS and measuring the absorbance of the solute at $570 \mathrm{~nm}$. From these dose-response curves, the $\mathrm{IC}_{50}$ of hydroxyurea in each cell line was estimated (dashed red line) and is presented in panel (c).

Figure 3(b)). Oleamide by itself was not toxic to either cell line. Interestingly, when GJIC was assessed in the oleamide treated WB-F344 cells, it was completely inhibited after 2 and $4 \mathrm{~h}$ of treatment, but then gradually returned to the control level by $24 \mathrm{~h}$ (Figure 3(c)). This indicates that during $48 \mathrm{~h}$ co-treatment with $\mathrm{HU}$ and oleamide, GJIC was completely inhibited only during the first $4 \mathrm{~h}$ and not at all during the last $24 \mathrm{~h}$.

\subsection{Effects of HU on Deoxynucleotide Pools}

Hydroxyurea had no differential effects on deoxynucleotide pools in GJIC-proficient and deficient cells (Figure 4). 


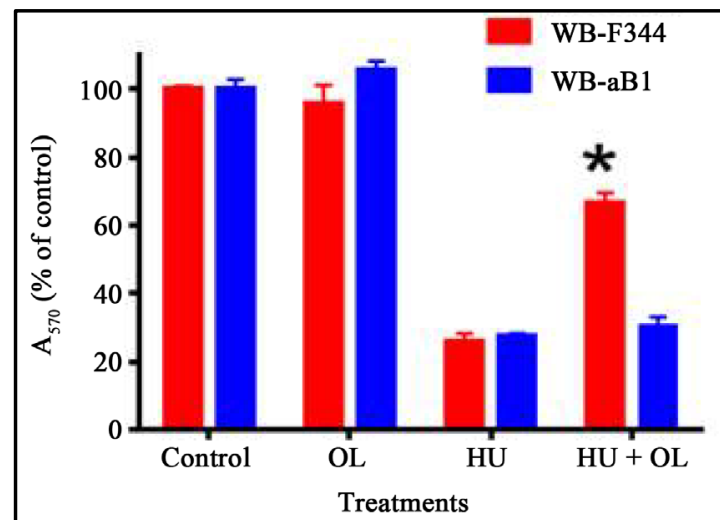

(a)

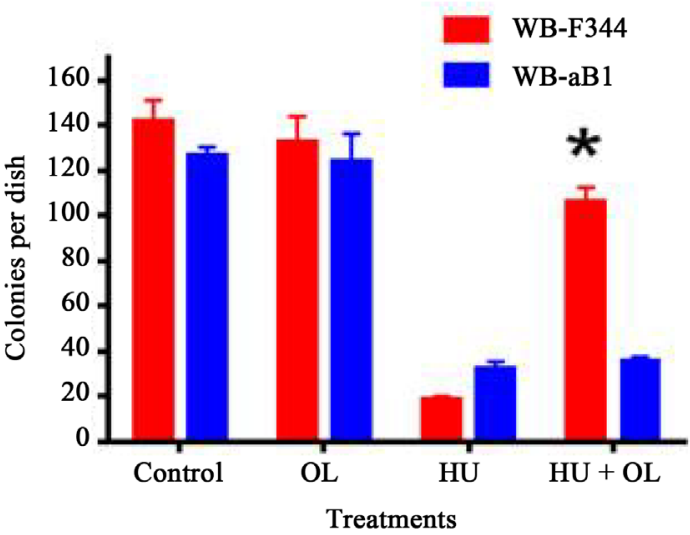

(b)

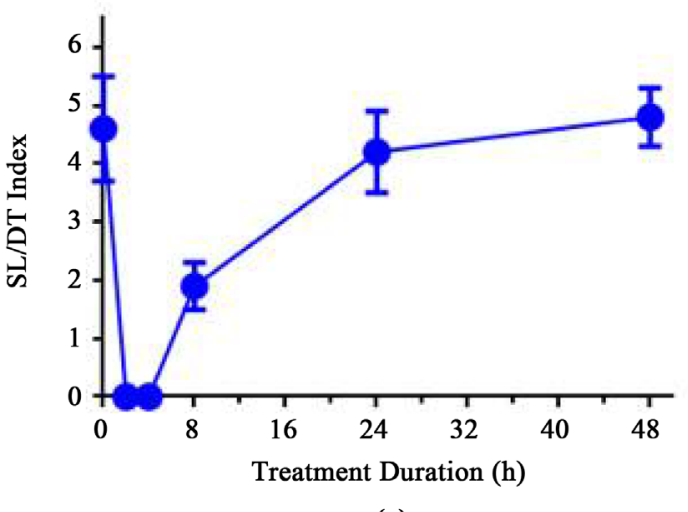

(c)

Figure 3. Effects of the GJIC inhibitor, oleamide, on hydoroxyurea (HU) cytotoxicity in WB-F344 and WB-aB1 cells. In panel (a), cytotoxicity was determined by crystal violet staining. The cells were cultured in 24 wells to $75 \%$ confluence, treated with or without oleamide $(50 \mu \mathrm{M})$ followed by $\mathrm{HU}$ at the $\mathrm{IC}_{75}$ concentration for each cell line $(0.125 \mathrm{mM}$ for WB-F344 cells, $0.5 \mathrm{mM}$ for WB-aB1 cells) for $48 \mathrm{~h}$, then washed, refed with complete medium, cultured for three days, and fixed and stained with crystal violet. Staining was quantified by solubilizing the residual cells in $2 \%$ SDS and measuring the absorbance of the solute at $570 \mathrm{~nm}$. In panel (b), cytotoxicity was assessed by colony formation assay. Cells were cultured in 24 wells to $75 \%$ confluence, treated with or without oleamide $(50 \mu \mathrm{M})$ followed by the $\mathrm{IC}_{75}$ concentration of HU for each cell line ( $0.125 \mathrm{mM}$ for WB-F344 cells, $0.5 \mathrm{mM}$ for WB-aB1 cells) for $48 \mathrm{~h}$, then washed and trypsinized. Two hundred cells were then plated into $35 \mathrm{~mm}$ dishes. After five days, the cultures were fixed and stained with crystal violet and colonies containing at least 50 cells were counted. The bars indicate the mean \pm S.D. ( $=4$ cultures per bar) and asterisks indicate significant differences $(\mathrm{p}<0.05)$ compared to cultures treated only with HU. In panel (c), the time-dependent inhibition of GJIC by oleamide in WB-F344 cells was evaluated by scrape-loading/dye transfer (SL/DT) assay. The points indicate the mean \pm S.D. $(n=4)$. 

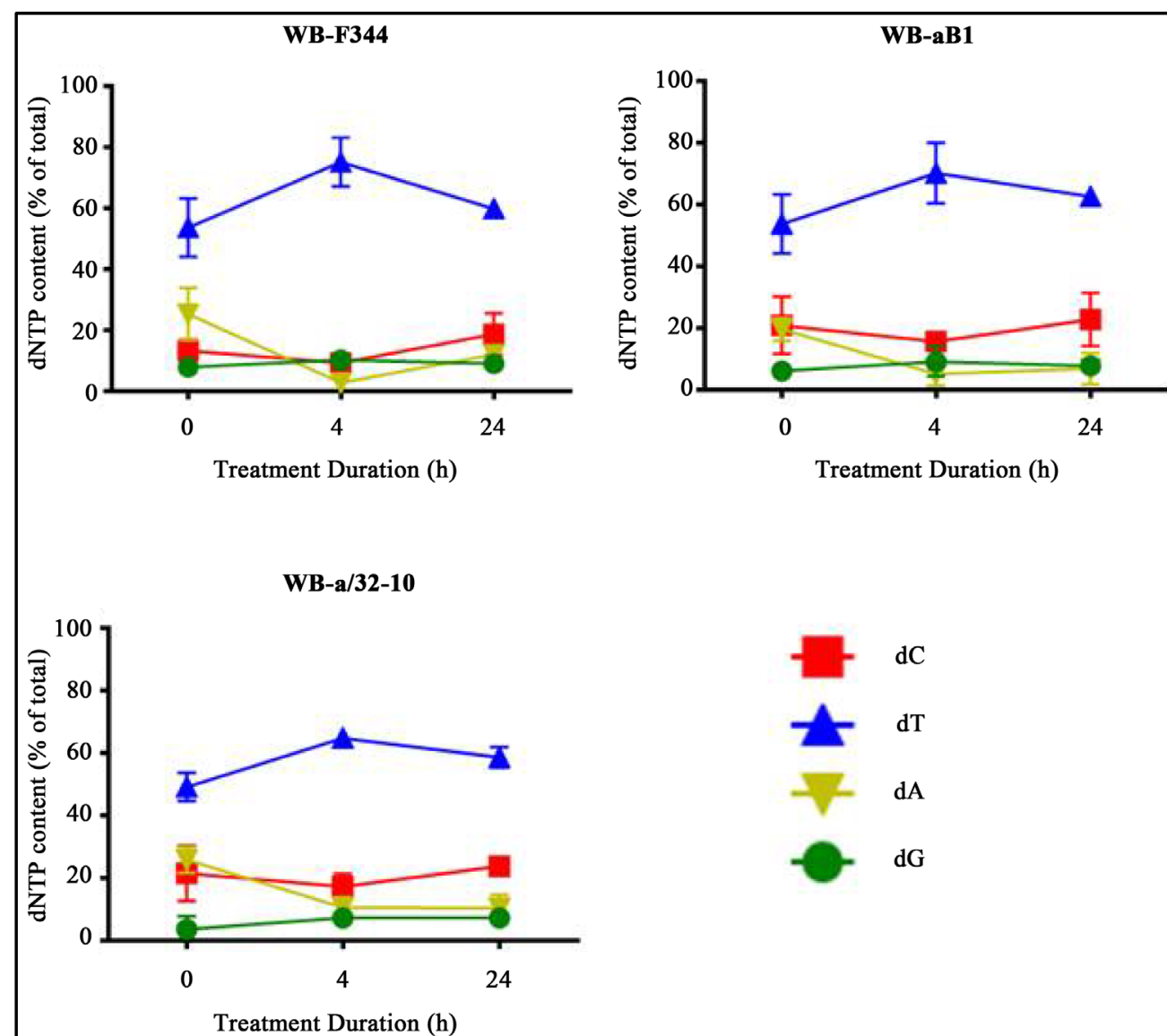

Figure 4. Deoxynucleotide content in WB-F344, WB-aB1, and WB-a/32-10 cells 0, 4, and 24 h after treatment with $\mathrm{IC}_{50}$ concentrations of hydroxyurea $(0.1,0.3$, and $0.1 \mathrm{mM}$, respectively). The deoxynucleotides were extracted from the cultures and quantified by anion exchange HPLC (mean \pm S.D.; $n$ $=8$ cultures per data point).

Pre-treatment levels of the four deoxynucleotides were similar in WB-F344, WB-aB1, and WB-a/32-10 cells. Following $4 \mathrm{~h}$ treatment with $\mathrm{HU}$ at the respective $\mathrm{IC}_{50}$ concentrations of each cell line, increases in thymidine triphosphate and decreases in deoxyadenosine triphosphate were seen. These changes were approximately $15 \%$ $20 \%$ and were similar in the three cell lines. After $24 \mathrm{~h}$ treatment, the levels of thymidine triphosphate had returned to pre-treatment concentrations whereas deoxyadenosine triphosphate levels remained decreased.

\subsection{GJIC Increases Cytotoxicity in HU-Resistant Cells}

Resistance to HU can occur in chronically treated cells in the laboratory and in cancer patients. HU resistant variants of WB-F34 and WB-aB1 cells were derived by stepwise increases in HU concentrations in the culture medium. Figure 5(a) shows the dose-response curves for these HU-resistant lines and their parental cells. Resistance to HU is typically associated with increased expression of RR-M2 [18] [19] and this was also the case for the resistant WB cells (Figure 5(b)). Baseline RR-M2 content in the two parental cell lines was comparable but dramatically increased in the resistant derivatives. GJIC was determined in the resistant cells by scrapeloading/dye transfer and did not differ from the parental cells. WB-F344/HU ${ }^{\mathrm{R}}$ exhibited robust dye transfer and WB-aB1/HU ${ }^{\mathrm{R}}$ showed no transfer (data not shown).

We next asked whether drug sensitivity in HU resistant cells was impacted by GJIC with drug sensitive cells. The HU-resistant cells were co-cultured with HU-sensitive, GJIC proficient WB-F344 cells at 1:3 ratios (resistant:sensitive) to increase contact of resistant cells with sensitive cells and treated with $1 \mathrm{mM} \mathrm{HU}$ for $48 \mathrm{~h}$. In these co-cultures, all of the HU-sensitive WB-F344 cells were killed (data not shown) as would be expected from the HU dose-response curve (Figure 5(a)). Importantly, GJIC-proficient WB-F344/HU ${ }^{\mathrm{R}}$ cells were also 


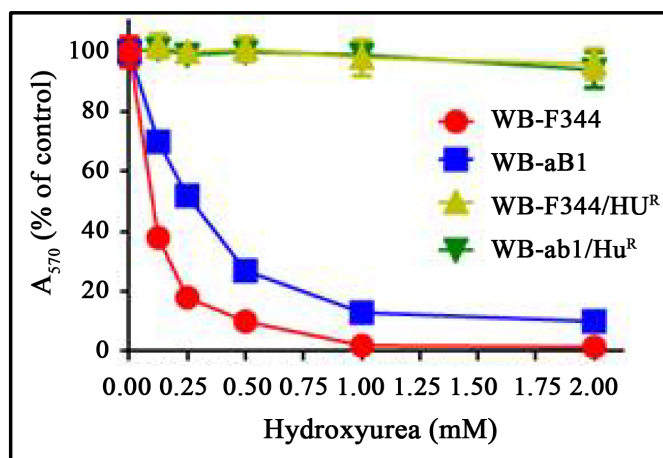

(a)

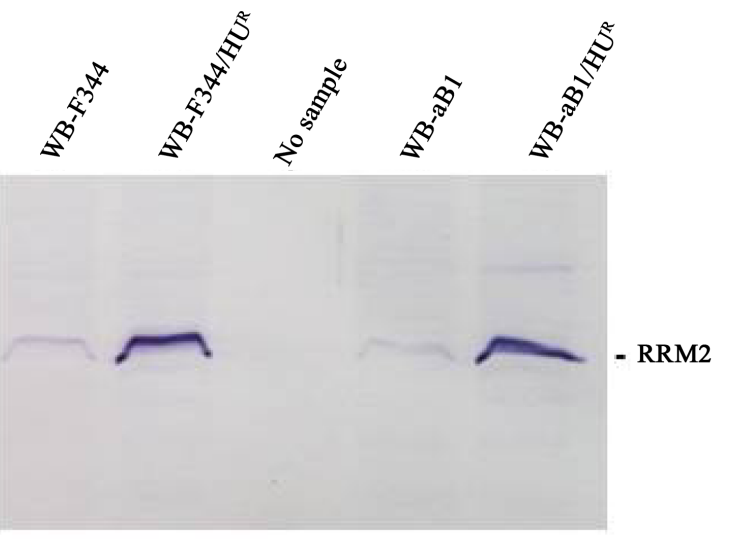

(b)

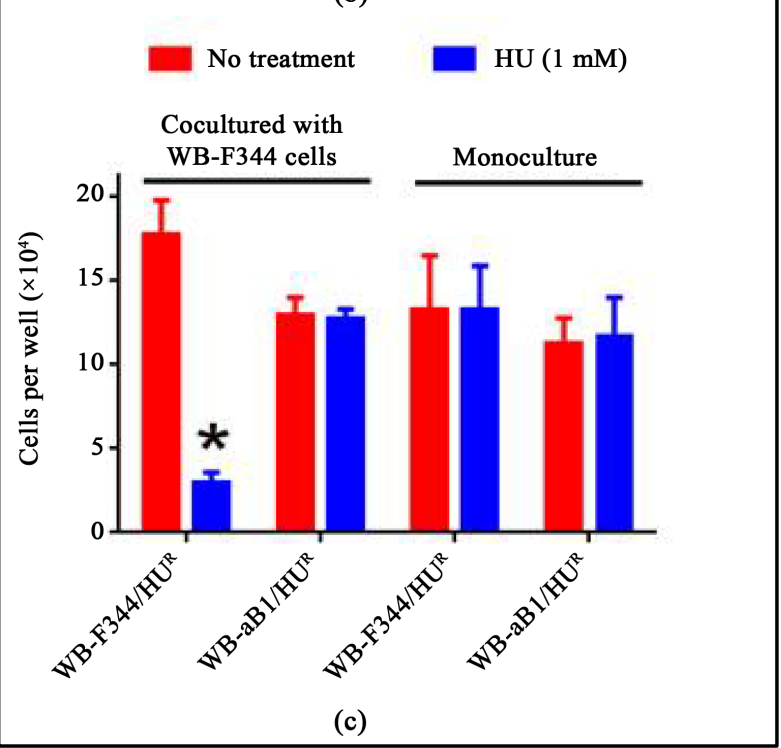

Figure 5. Characterization of hydroxyurea-resistant WB-F344/HU $\mathrm{U}^{\mathrm{R}}$ and $\mathrm{WB}-\mathrm{aB} 1 / \mathrm{HU}^{\mathrm{R}}$ cells and the effects of co-culture with hydroxyurea-sensitive WB-F344 cells on this resistance. Panel (a) illustrates the toxicity of hydroxyurea in the resistant cells and their parental cells, WB-F344 and WB-aB1, respectively, determined by crystal violet staining. Panel (b) is a western blot of the M2 subunit of ribonucleotide reductase (RR-M2) in these resistant and sensitive cells. In panel (c), the effects of co-culture with sensitive WB-F344 cells on hydroxyurea toxicity in the resistant cells is shown. The resistant cells were co-cultured with WB-F344 cells at a 1:3 ratio and treated with $1 \mathrm{mM}$ hydroxyurea for $48 \mathrm{~h}$. Then the remaining cells were trypsinized and counted. The resistant cells were labeled with PKH-26 vital fluorescent dye before co-culture to distinguish them from WB-F344 cells. The bars indicate the mean \pm S.D. ( $n=4$ cultures per bar) and the asterisk indicates a significant difference $(\mathrm{p}<0.05)$ compared to the no treatment group. 
killed extensively, but GJIC-deficient WB-aB1/HU ${ }^{\mathrm{R}}$ cells were not (Figure 5(c), left side of graph). As controls, the resistant cells were cultured alone at the same plating density as in the co-cultures and no HU toxicity occurred (Figure 5(c), right side of graph). Thus resistance to HU is decreased when resistant cells are co-cultured with sensitive cells and GJIC is present. Under the experimental conditions, it was also apparent that the resistant cells did not protect the sensitive cells.

\section{Discussion}

Here we have demonstrated that cells that are well-coupled by gap junctions are more sensitive to the classic RR inhibitor and anti-cancer agent, $\mathrm{HU}$. Based on the $\mathrm{IC}_{50}$ values for $\mathrm{HU}$ in each cell line, this increased sensitivity was approximately three to fivefold. This was seen whether the cells were coupled by gap junctions comprised of Cx43 or Cx32. We also showed the effect was dependent upon GJIC and not due to differences in connexin expression or localization. The GJIC-deficient cells expressed comparable amounts of Cx43 that was localized to the plasma membrane similar to the more sensitive, GJIC-proficient parental cells (Figure 1) [16] [17]. The effects were also not related to rates of cell proliferation (Figure 1).

Several groups have also observed increased cytotoxicity of cancer cells to a variety of chemotherapeutic agents such as doxorubicin, cisplatin, vinblastine, docetaxel, and BCNU in association with GJIC (reviewed in [5]). These enhanced chemotherapeutic drug effects were associated with reduced expression of bcl-2 and increased apoptosis, decreased expression of p-glycoprotein, lowered intracellular glutathione content, and gap junction-mediated transfer of apoptosis-inducing signals from damaged cells to neighbors. These results are reminiscent of the GJIC-mediated propagation and amplification of cell death in glial cells during cerebral ischemia [20]. Similarly, GJIC contributes to the radiation "bystander effect" in which signaling molecules and/or reactive oxygen species induce damage in neighboring, non-irradiated cells [21] [22]. In contrast, GJIC may also reduce drug, ischemic, and radiation-induced cytotoxicity possibly by cell-cell sharing of protective molecules like glutathione [5] [23]. Furthermore, these effects may not be due solely to GJIC. Transfection of cancer cells with non-gap junction-forming, mutant connexins also induced similar phenotypic and cytotoxic changes. External factors released from damaged cells, in some cases via gap junction hemichannels, also contribute to these bystander effects [5]. Thus, gap junctions and connexins may affect cell toxicity in several ways.

Our study is the first to report that GJIC increases the cytotoxicity of HU. This drug is a potent inhibitor of $\mathrm{RR}$ which is active during $\mathrm{S}$ phase and converts ribonucleotides to deoxyribonucleotides [6] [7]. The inhibition of RR causes imbalances in dNTP pools that lead to nucleotide misincorporation into DNA, S phase arrest, and apoptosis. Cytotoxic effects are greatest in proliferating cells, but RR inhibitors also kill non-proliferating cells. The mechanisms for non-proliferating cell toxicity are not clear, but may also be related to nucleotide pool imbalances.

Our data do not suggest the differential toxicity is due to differences in dNTP pool imbalances. We detected modest $(15 \%-20 \%)$ increases in thymidine triphosphate pools and similar decreases in deoxyadenosine triphosphaye pools $4 \mathrm{~h}$ after treatment of WB cells with HU. The responses were similar in GJIC-proficient and deficient cells and were not related to rates of cell proliferation. We also noted similar baseline levels of RR-M2 expression in these cells and comparable enhanced expression of the subunit in HU-resistant cells. These results suggest that GJIC-proficient and deficient cells respond similarly to HU in terms of its actions on RR and nucleotide pool imbalances and that other factor(s) may account for GJIC-dependent enhanced toxicity. HU is also metabolized to free radical intermediates such as nitric oxide that cause oxidative stress, cell damage, and death [24]. It is possible that GJIC-proficient and deficient WB cells differ in the metabolism of HU or in their detoxification/antioxidant capacity.

It is worth considering whether a connexin-mediated, non-GJIC dependent mechanism could be involved in this differential toxicity. Many reports have demonstrated non-GJIC dependent effects of Cx43 and other connexins on cellular functions and drug repsonses. Signaling and structural proteins interact with cytoplasmic domains of connexins so that the latter are considered components of cellular adhesion and signal transduction pathways [4]. Connexins or fragments of them have also been localized in the nucleus and may function in gene regulation [13] [25]. Our results suggest, however, that the enhancement of HU toxicity in GJIC-proficient WB cells was not due to differences in $\mathrm{Cx} 43$ expression per se, its localization, or a non-GJIC mechanism. First, $\mathrm{Cx} 43$ protein content and plasma membrane localization are comparable in the cell lines we have used (Figure 1) [16] [17]. Second, oleamide which blocks GJIC, but does not affect expression or localization [8], reduced HU 
toxicity only in GJIC-proficient cells. Third, restoration of GJIC by stable Gjb1 (Cx32) transduction of WB-aB1 cells increased HU toxicity. Lastly, it is unlikely that the release of toxic or other factors from gap junction hemichannels was involved since GJIC-deficient, HU-resistant cells were not killed by co-cultured HU-sensitive cells. Instead, the common determinant in the enhanced cytoxicity of HU was the presence of GJIC.

This enhancement of HU toxicity may have occurred within the first few hours of HU exposure. Blockage of GJIC with oleamide greatly reduced HU toxicity during a $48 \mathrm{~h}$ co-exposure experiment, but blockage was transient with complete blockage over the first $4 \mathrm{~h}$ of treatment followed by recovery to control levels within $24 \mathrm{~h}$. This suggests that whatever GJIC-dependent molecular information is involved in the differential toxicity to HU, it is likely conveyed within the first few hours of treatment.

There are 21 human and 20 mouse connexin genes and cells typically express at least two types [1] [2]. Gap junction hemichannels can be homomeric or heteromeric and complete channels can be homotypic (identical hemichannels) or heterotypic (different hemichannels) depending upon connexin expression and hemichannel composition of the apposed cells. Differences in channel permeability, regulation of gating, and association with other proteins have been noted depending upon the connexin composition of the channels [3] [4]. Because these variations impact the types of molecules that move between cells through gap junction channels, they likewise may impact GJIC-dependent toxicity. We have only detected Cx43 in the WB cell lines indicating this connexin can mediate enhanced cytotoxicity of HU effect in WB cells. However, similar effects were seen when GJIC was restored in WB-aB1 cells by Gjb1-transduction. Thus, GJIC mediated by both Cx43 and Cx32 channels can enhance HU toxicity in WB cells.

Finally, our data demonstrate a potential clinically important result. When HU-resistant cells were co-cultured with HU-sensitive cells, drug resistance was overcome, but only when GJIC was present. This suggests bystander killing of HU-resistant cells by the drug they are resistant to can be achieved through GJIC. The mechanism(s) for this remain to be determined. Possibilities include GJIC-mediated transfer of cytotoxic signal molecules or other factors from sensitive cells to resistant cells, reduction of protective molecules in resistant cells by GJIC with sensitive ones, and epigenetic changes in sensitive cells induced by GJIC. Importantly, in a clinical setting, resistance to an anticancer drug may be reduced or overcome if drug-resistant cells can be induced to form gap junctions. This may be especially relevant to cancer stem cells which often are resistant to cancer treatments. Cancer stem cells have not been well studied for connexin expression and GJIC; one report indicated glioma stem cells were deficient in connexin 43 expression [26]. Several agents increase GJIC in neoplastic cells and may be beneficial in these approaches [27].

\section{Conclusion}

The data show that GJIC enhances cytotoxicity and decreases resistance to HU in WB cells. This was mediated by gap junctions containing connexin 43 or connexin 32. The result may be clinically relevant if GJIC can be enhanced in drug-resistant tumor cells including cancer stem cells that may lack GJIC.

\section{Acknowledgements}

Supported by NIH/NCI grants CA117242, CA076581, and CA083081.

\section{References}

[1] Evans, W.H. and Martin, P.E. (2002) Gap Junctions: Structure and Function (Review). Molecular Membrane Biology, 19, 121-136. http://dx.doi.org/10.1080/09687680210139839

[2] Sohl, G. and Willecke, K. (2003) An Update on Connexin Genes and Their Nomenclature in Mouse and Man. Cell Communication and Adhesion, 10, 173-180. http://dx.doi.org/10.1080/cac.10.4-6.173.180

[3] Dbouk, H.A., Mroue, R.M., El-Sabban, M.E. and Talhouk, R.S. (2009) Connexins: A Myriad of Functions Extending beyond Assembly of Gap Junction Channels. Cell Communication and Signaling, 7, 1-17. http://dx.doi.org/10.1186/1478-811X-7-4

[4] Herve, J.C., Bourmyster, N., Sarrouilhe, D. and Duffy, H.S. (2007) Gap Junctional Complexes: From Partners to Functions. Progress in Biophysics \& Molecular Biology, 94, 29-65. http://dx.doi.org/10.1016/j.pbiomolbio.2007.03.010

[5] Decrock, E., Vinken, M., De Vuyst, E., Krysko, D.V., D’Herde, K., Vanhaecke, T., Vandenabeele, P., Rogiers, V. and Leybaert, L. (2009) Connexin-Related Signaling in Cell Death: To Live or Let Die? Cell Death and Differentiation, 16, 151-163. http://dx.doi.org/10.1038/cdd.2008.138 
[6] Nocentini, G. (2006) Ribonucleotide Reductase Inhibitors: New Strategies for Cancer Chemotherapy. Critical Reviews in Oncology and Hematology, 22, 89-126. http://dx.doi.org/10.1016/1040-8428(95)00187-5

[7] Navarra, P. and Preziosi, P. (1999) Hydroxyurea: New Insights on an Old Drug. Critical Reviews in Oncology and Hematology, 29, 249-255. http://dx.doi.org/10.1016/S1040-8428(98)00032-8

[8] Guan, X., Cravatt, B.F., Ehring, G.R., Hall, J.E., Boger, D.L., Lerner, R.A. and Gilula, N.B. (1997) The Sleep-Inducing Lipid Oleamide Deconvolutes Gap Junction Communication and Calcium Wave Transmission in Glial Cells. Journal of Cell Biology, 139, 1785-1792. http://dx.doi.org/10.1083/jcb.139.7.1785

[9] Esinduy, C.B., Chang, C.C., Trosko, J.E. and Ruch, R.J. (1995) In Vitro Growth Inhibition of Neoplastically Transformed Cells by Non-Transformed Cells: Requirement for Gap Junctional Intercellular Communication. Carcinogenesis, 16, 915-921. http://dx.doi.org/10.1093/carcin/16.4.915

[10] Boucher, P.D., Ostruszka, L.J. and Shewach, D.S. (2000) Synergistic Enhancement of Herpes Simplex Virus Thymidine Kinase/Ganciclovir-Mediated Cytoxicity by Hydroxyurea. Cancer Research, 60, 1631-1636.

[11] El-Fouly, M.H., Trosko, J.E. and Chang, C.C. (1987) Scrape-Loading and Dye Transfer: A Rapid and Simple Technique to Study Gap Junctional Intercellular Communication. Experimental Cell Research, 168, 422-430. http://dx.doi.org/10.1016/0014-4827(87)90014-0

[12] Nielsen, M., Ruch, R.J. and Vang, O. (2000) Resveratrol Reverses Tumor-Promoter-Induced Inhibition of Gap-Junctional Intercellular Communication. Biochemical and Biophysical Research Communications, 275, 804-809. http://dx.doi.org/10.1006/bbrc.2000.3378

[13] Guan, X.J. and Ruch, R.J. (1996) Gap Junction Endocytosis and Lysosomal Degradation of Connexin 43-P2 in WBF344 Rat Liver Epithelial Cells Treated with DDT and Lindane. Carcinogenesis, 17, 1791-1798. http://dx.doi.org/10.1093/carcin/17.9.1791

[14] Mehta, P.P., Lokeshwar, B.L., Schiller, P.C., Bendix, M.V., Ostenson, R.C., Howard, G.A. and Roos, B.A. (1996) Gap-Junctional Communication in Normal and Neoplastic Prostate Epithelial Cells and Its Regulation by cAMP. Molecular Carcinogenesis, 15, 18-32. http://dx.doi.org/10.1002/(SICI)1098-2744(199601)15:1<18::AID-MC4>3.0.CO;2-O

[15] Tsao, M.S., Smith, J.D., Nelson, K.G. and Grisham, J.W. (1984) A Diploid Epithelial Cell Line from Normal Adult Rat Liver with Phenotypic Properties of “Oval” Cells. Experimental Cell Research, 154, 38-52. http://dx.doi.org/10.1016/0014-4827(84)90666-9

[16] Oh, S.Y., Dupont, E., Madhukar, B.V., Briand, J.P., Chang, C.C., Beyer, E. and Trosko, J.E. (1993) Characterization of Gap Junctional Communication-Deficient Mutants of a Rat Liver Epithelial Cell Line. European Journal of Cell Biology, 60, 250-255.

[17] Rae, R.S., Mehta, P.P., Chang, C.C., Trosko, J.E. and Ruch, R.J. (1998) Neoplastic Phenotype of Gap-Junctional Intercellular Communication-Deficient WB Rat Liver Epithelial Cells and Its Reversal by Forced Expression of Connexin 32. Molecular Carcinogenesis, 22, 120-127. http://dx.doi.org/10.1002/(SICI)1098-2744(199806)22:2<120::AID-MC7>3.0.CO;2-Q

[18] Jiang, R., Zhang, J.L., Satoh, Y. and Sairenji, T. (2004) Mechanism for Induction of Hydroxyurea Resistance and Loss of Latent EBV Genome in Hydroxyurea-Treated Burkitt’s Lymphoma Cell Line Raji. Journal of Medical Virology, 73, 589-595. http://dx.doi.org/10.1002/jmv.20130

[19] Yen, Y., Grill, S.P., Dutschman, G.E., Chang, C.N., Zhou, B.S. and Cheng, Y.C. (1994) Characterization of a Hydroxyurea-Resistant Human KB Cell Line with Supersensitivity to 6-Thioguanine. Cancer Research, 54, 3686-3691.

[20] Lin, J.H., Weigel, H., Cotrina, M.L., Liu, S., Bueno, E., Hansen, A.J., Hansen, T.W., Goldman, S. and Nedergaard, M. (1998) Gap-Junction-Mediated Propagation and Amplification of Cell Injury. Nature Neuroscience, 1, 494-500. http://dx.doi.org/10.1038/2210

[21] Hamada, N., Matsumoto, H., Hara, T. and Kobayashi, Y. (2007) Intercellular and Intracellular Signaling Pathways Mediating Ionizing Radiation-Induced Bystander Effects. Journal of Radiation Research, 48, 87-95. http://dx.doi.org/10.1269/jrr.06084

[22] Hei, T.K., Zhou, H., Chai, Y., Ponnaiya, B. and Ivanov, V.N. (2011) Radiation Induced Non-Targeted Response: Mechanism and Potential Clinical Implications. Current Molecular Pharmacology, 4, 96-105. http://dx.doi.org/10.2174/1874467211104020096

[23] Nakase, T., Fushiki, S. and Naus, C.C. (2003) Astrocytic Gap Junctions Composed of Connexin 43 Reduce Apoptotic Neuronal Damage in Cerebral Ischemia. Stroke, 34, 1987-1993. http://dx.doi.org/10.1161/01.STR.0000079814.72027.34

[24] King, S.B. (2003) The Nitric Oxide Producing Reactions of Hydroxyurea. Current Medicinal Chemistry, 10, 437-452. http://dx.doi.org/10.2174/0929867033368213

[25] Mennecier, G., Derangeon, M., Coronas, V., Hervé, J.C. and Mesnil, M. (2008) Aberrant Expression and Localization 
of Connexin 43 and Connexin 30 in a Rat Glioma Cell Line. Molecular Carcinogenesis, 47, 391-401. http://dx.doi.org/10.1002/mc.20393

[26] Yu, S.C., Xiao, H.L., Jiang, X.F., Wang, Q.L., Li, Y., Yang, X.J., Ping, Y.F., Duan, J.J., Jiang, J.Y., Ye, X.Z., Xu, S.L., Xin, Y.H., Yao, X.H., Chen, J.H., Chu, W.H., Sun, W., Wang, B., Wang, J.M., Zhang, X. and Bian, X.W. (2012) Connexin 43 Reverses Malignant Phenotypes of Glioma Stem Cells by Modulating E-Cadherin. Stem Cells, 30, 108-120. http://dx.doi.org/10.1002/stem.1685

[27] Trosko, J.E. and Ruch, R.J. (2002) Gap Junctions as Targets for Cancer Prevention and Chemotherapy. Current Drug Targets, 3, 465-482. http://dx.doi.org/10.2174/1389450023347371 
Scientific Research Publishing (SCIRP) is one of the largest Open Access journal publishers. It is currently publishing more than 200 open access, online, peer-reviewed journals covering a wide range of academic disciplines. SCIRP serves the worldwide academic communities and contributes to the progress and application of science with its publication.

Other selected journals from SCIRP are listed as below. Submit your manuscript to us via either submit@scirp.org or Online Submission Portal.
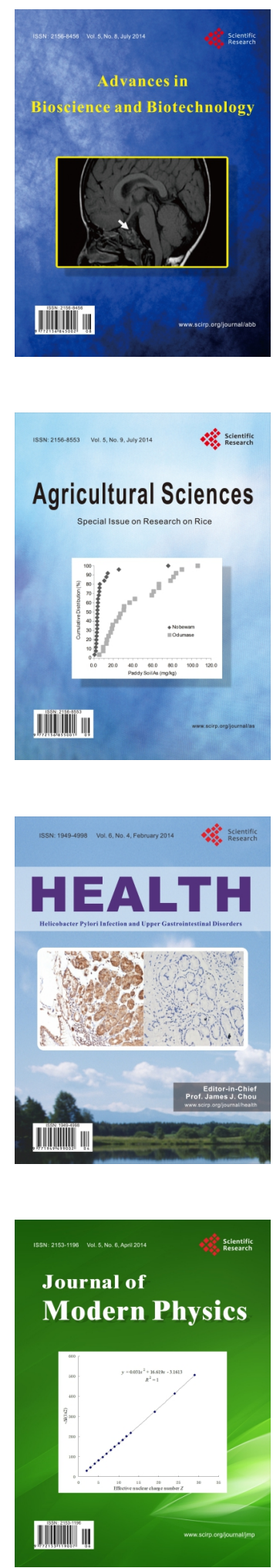
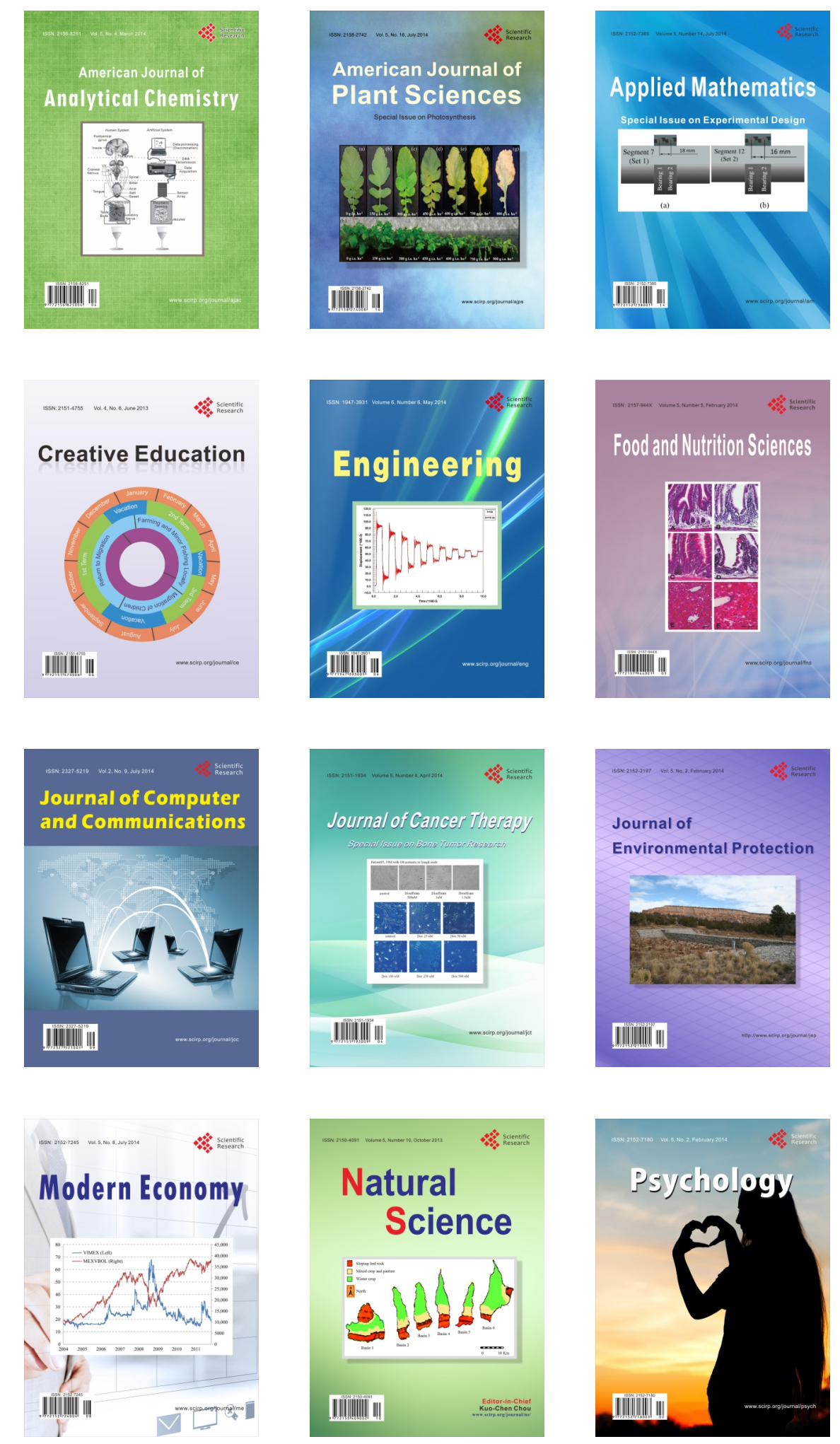